\title{
MS18-06 | INCREASE YOUR ENERGY WITH XRD
}

Welzmiller, Simon (Thermo Fisher Scientific, Ecublens, $\mathrm{CH}$ )

Due to humanity's growing use of energy, it is crucial to enhance the capabilities in both, production and storage of environmentally sustainable electricity. The usage of the sun as unlimited power source requires the design of highly efficient photovoltaic materials to convert light into usable electrical energy. Currently there are three predominantly used materials for thin film solar cells (Cadmium telluride CdTe, amorphous silicon, Copper Indium Gallium Telluride CIGS). Such solar cells are more cost efficient compared to bulk solar cells (crystalline silicon) as the required amount of material is very low. Nevertheless, they still exhibit lower cell efficiencies due to less mature upscaling compared to crystalline silicon based solar cells. Anyways, the latter materials are still intensively investigated to enhance their properties, usually by chemical substitution and designing the interfaces of the solar cell layers.

The crystallographic structure of multi-functional inorganic and hybrid organic-inorganic thin films (nano-meter scale) has to be characterized by GIXRD (grazing incidence XRD). This is important as electronical and optical properties strongly depend on the structure of compounds.

Another variable in designing thin film materials for various applications is the thickness, roughness and density of certain layers. One technique for determining these properties in (multi)-layers up to $\sim 100 \mathrm{~nm}$ is $\mathrm{X}$-ray reflectometry (XRR), which is based on the interference between X-rays reflected on different layers in the material. In contrast to XRR, which requires perfectly homogeneous samples, X-ray fluorescence spectroscopy (XRF) is also able to determine the thickness of (multi)-layer films by using a fundamental parameter approach in both wavelength-dispersive and energy-dispersive XRF. 